\title{
ANÁLISIS DE LAS SITUACIONES DE INCLUSIÓN Y RESPUESTA EDUCATIVA DE ALUMNOS INMIGRANTES EN DOS CENTROS EDUCATIVOS DE LA COMUNIDAD AUTONOMA DE MADRID
}

\author{
Ana Maria González TABERNERO ${ }^{1}$ \\ Verónica Cerrillo CALLEJA ${ }^{2}$ \\ Eladio Sebastián HEREDERO ${ }^{3}$
}

RESUMEN: Este trabajo de investigación está basado en la inmigración en la escuela. Debido a que en los últimos años el número de alumnado extranjero se ha incrementando notablemente. La investigación ha sido realizada en dos centros educativos pertenecientes al Corredor del Henares, más concretamente en cuatro clases de diferentes niveles de educación primaria. Estos colegios presentan grandes diferencias en el porcentaje de alumnado extranjero matriculado. Al realizar este trabajo nos hemos centrado en los siguientes aspectos a estudiar: en primer lugar, las relaciones sociales con la finalidad de conocer el grado de integración de los alumnos extranjeros con los sus compañeros de clase. A continuación, hemos investigado las medidas adoptadas por los dos centros para la integración y adaptación de estos alumnos al centro escolar y al sistema educativo. Y finalmente hemos analizado como se ajustan esta medidas a la situación de los alumnos extranjeros.

PALABRAS CLAVE: Inclusión. Integración. Adaptación curricular. Plan de trabajo individualizado. Apoyos. Medidas atención a la diversidad. Inmigración. Aula de enlace.

\section{Introducción}

Hemos elegido el tema de la inmigración en la escuela, debido a los continuos cambios que se han ido produciendo en los últimos años tanto en materia de gran incremento de su número como en la forma y calidad de la respuesta. Es un tema que como profesores nos interesa y debemos estar informados para hacer lo posible por la integración en nuestras aulas de este alumnado

Al principio, la investigación ha sido enfocada desde el contexto global, la Comunidad Autónoma $\left(\mathrm{CCAA}^{4}\right)$ de Madrid para más tarde centrarnos en la realidad más particular de dos centros educativos del Corredor de Henares. Para ello, hemos investigado sobre la inmigración en la CCAA de Madrid y la respuesta educativa de la misma. Hemos incluido los

\footnotetext{
${ }^{1}$ Alumna de $3^{\circ}$ Magisterio. Universidade de Alcalá. Escola Universitária de Magisterio. Alcalá de Henares Espanha. 28801

2 Alumna de $3^{\circ}$ Magisterio. Universidade de Alcalá. Escola Universitária de Magisterio. Alcalá de Henares Espanha. 28801 - veronicacerrillo@gmail.com

3 Universidad de Alcalá. Departamento de Didáctica. Alcalá de Henares - Espanha. 28801 eladio.sebastian@uah.es

${ }^{4}$ Las Comunidades Autónomas españolas tiene transferidas todas las competencias en materia de educación desde el año 2000 funcionando cada una de ellas de forma autónoma por lo que pueden, y de hecho se organizan medidas y recursos diferentes en cada una de ellas.
} 
servicios que ofrece la Conserjería de Educación para informarnos después de la utilización de los mismos en los centros.

\section{Inmigración en la comunidad de Madrid}

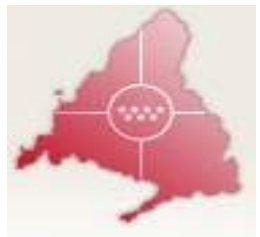

Durante las últimas décadas España ha sido uno de los países de la Unión Europea, que más flujo de inmigrantes ha recibido y Madrid, como capital, es una de las comunidades que más inmigración acoge. Actualmente, según el Informe de Población Extranjera Empadronada, en la región el número de población inmigrante en 2008, se elevo a 1.108 .920 habitantes, lo que representa el 17,12 \% del total de la población madrileña. En cuanto a las nacionalidades, es la rumana la mayoritaria en la Comunidad, seguida de la ecuatoriana, la marroquí, colombiana y peruana.

\section{La Educación de la población inmigrante en la comunidad de Madrid}

Si nos centramos en esta CCAA, según la Consejería de Educación, el número de alumnos inmigrantes escolarizados en el curso 2008/2009 se ha incrementado un $10 \%$ con respecto al año anterior hasta alcanzar los 149.904 estudiantes llegados de fuera, una cifra que supone que el 16\% del alumnado madrileño es de origen extranjero. Según datos de la Consejería de Educación, las cuatro nacionalidades más representadas en el alumnado extranjero que estudia en la CCAA son Ecuador (32.695 alumnos), Rumania (17.933), Marruecos (14.191) y Colombia (11.087). Si observamos el número de alumnos de inmigrantes en los últimos cinco años veremos un crecimiento de los alumnos inmigrantes en las aulas españolas:

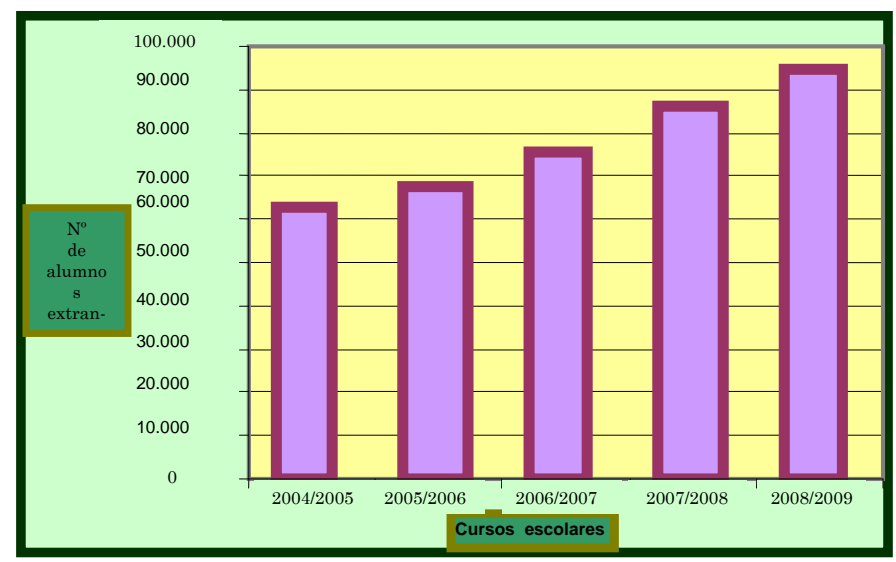

Tabla1 - Alumnado extranjero en la Comunidad de Madrid en los últimos cinco años. Fuente: Consejería de Educación (2008). 
Estos datos nos hacen recapacitar sobre las ayudas e intervenciones que deben dar los centros escolares para afrontar los posibles problemas que presenten algunos de los inmigrantes que inician el nuevo curso académico. Así, la Conserjería de Educación ha fortalecido los programas complementarios como el Servicio de Apoyo Itinerante al Alumnado Inmigrante, el Servicio de Traductores e Intérpretes (SETI) y las aulas de enlace.

\section{La población inmigrante en el corredor del henares}

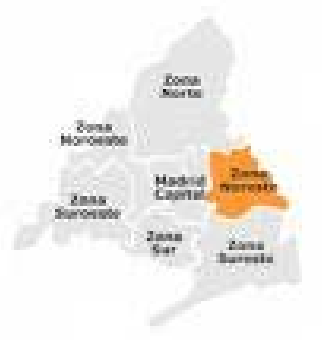

Desde el año 2000, el Corredor del Henares ha recibido de forma continuada población principalmente procedente de los países del Este de Europa, seguido de los países extracomunitarios, latinoamericanos y africanos.

En el año 1988 contaba con 8.880 habitantes, en el año 2006 había un total de 80.653 habitantes. Siendo un 50\% de la población inmigrante procedente de Rumania, un 25\% de Ecuador y Colombia y un 10\% de Marruecos. En cuanto al género es casi igual hay una cierta tendencia a la homogeneidad. Aunque si es cierto, que es mayor el porcentaje de nacimientos de madres extranjeras.

Como vemos en la tabla 2 Paracuellos del Jarama es el municipio que cuenta con el mayor porcentaje de población extranjera (17,59\%), seguido de Alcalá de Henares con un 17, 55\%, de Torrejón de Ardoz con un 17,35\% y Coslada con un 12,22 \% y por último San Fernando de Henares con 13,13 \%. Cabe decir, que a pesar de que

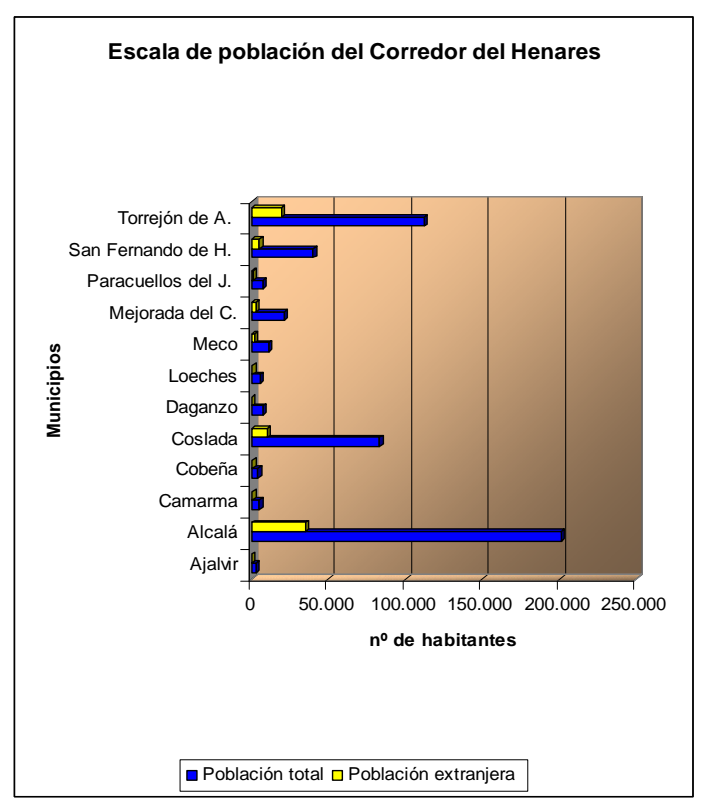
Paracuellos del Jarama tiene una población inferior al resto de los municipios citados anteriormente, es el que contiene un mayor porcentaje de población, ya que cuenta con un número elevado de inmigración respecto a su población total (1134 y 7.586 respectivamente ). Sin embargo, podemos destacar que el municipio que menos inmigrantes posee es Daganzo con un 6,32 \%, siguiéndole Ajalvir con 9,87\% y Camarma con un 10,12 \%.

La investigación que explicaremos a continuación, la hemos realizado en los municipios de San Fernando de Henares y Torrejón de Ardoz, ya que ambas localidades 
contienen un alto porcentaje de población inmigrante y por ello hemos querido reflejar como influye el contexto sociocultural de cada localidad en las escuelas.

En las siguientes gráficas se observan el claro predominio de la nacionalidad rumana en San Fernando de Henares y la sudamericana en Torrejón de Ardoz.
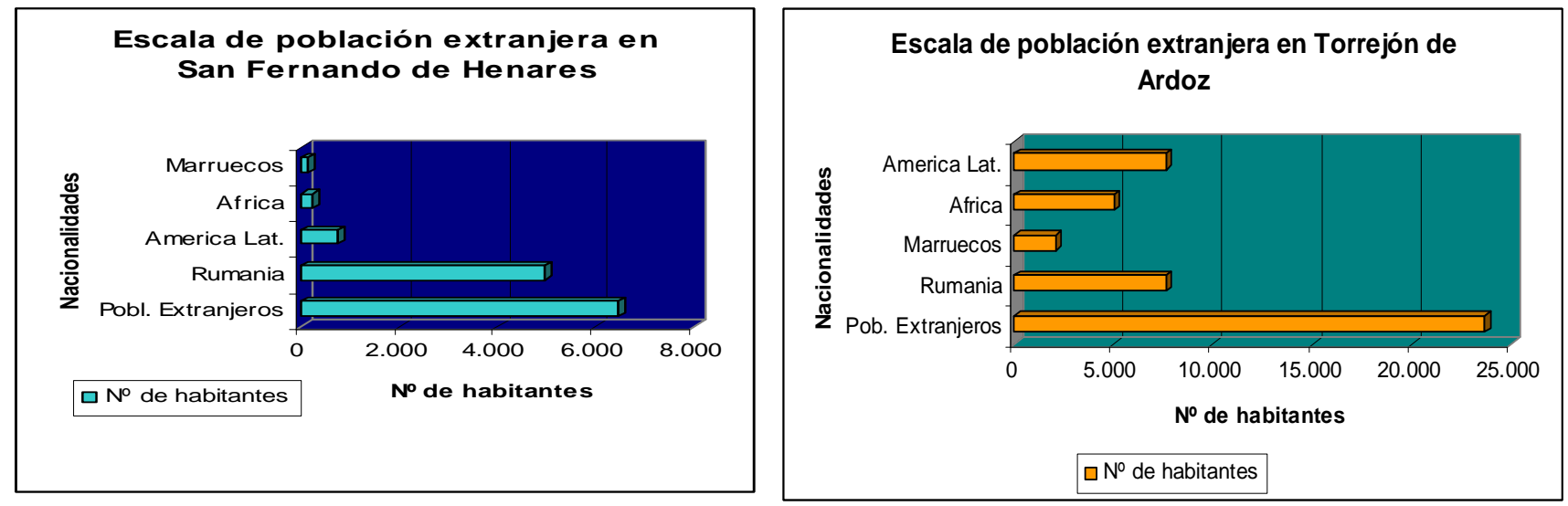

\section{Algunos problemas que presenta la población inmigrante}

Según un estudio realizado ${ }^{5}$ la mayoría de las personas inmigrantes consideran que la integración de los hijos en el plano educativo es buena, no obstante, hay un número menor de padres inmigrantes consideran que sus hijos son discriminados por sus compañeros de clase simplemente por su lugar de origen.

Si hablamos sobre la percepción que los inmigrantes tienen de los españoles, esta es bastante positiva, e incluso manifiestan haber recibido ayuda por parte de ellos. Aunque, también se percibe la existencia de sectores xenófobos que infravaloran a las personas inmigrantes.

Otro de los problemas que presenta la población inmigrante es el desconocimiento del Idioma, cuya consecuencia es la necesidad de un rápido aprendizaje del idioma español. Se destaca, el considerable número de inmigrantes procedentes de los países del Este de Europa y de África que no dominan bien el idioma a pesar de llevar viviendo varios años en España, este problema es transmitido a sus hijos y a su vez al colegio, ya que al ser bilingües sin un refuerzo en casa estos niños tienen frecuentes problemas en el aprendizaje.

\section{Estudio del contexto socio-cultural de los Colegios investigados}

- C.E.I.P Severo Ochoa (Torrejón de Ardoz)

\footnotetext{
${ }^{5}$ Cr Informe CIDE-MEC (2005)
}

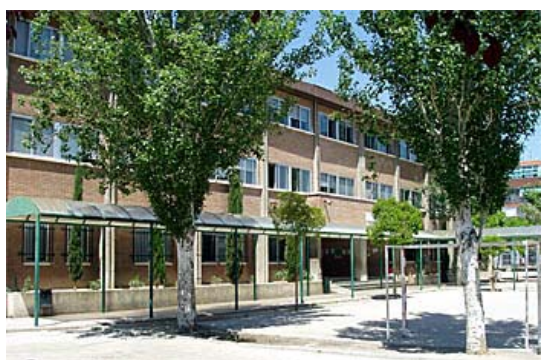


Está ubicado en el barrio de "Las Veredillas", en este barrio conviven nuevas zonas residenciales con otras más antiguas. El continuo crecimiento de Torrejón de Ardoz ha provocado la necesidad de construir nuevos bloques de pisos como sigue ocurriendo actualmente. Desde el punto de vista sociológico es una zona bastante asentada en donde encontramos familias que han vivido en este mismo municipio durante varias generaciones, y conviven con la llegada de otras familias formadas por jóvenes con un menor poder nivel adquisitivo y por inmigrantes. En términos generales, podríamos encuadrar a la población dentro de una clase media con un nivel económico estable.

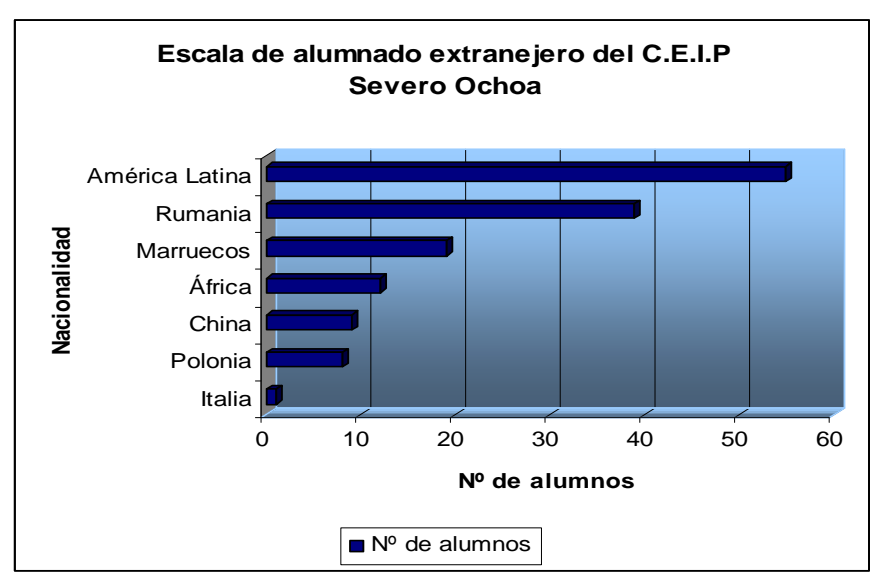

La tabla adjunta muestra la totalidad del número de alumnos extranjeros en este colegio de Torrejón de Ardoz. Este centro escolar cuenta con un elevado número de alumnos extranjeros. Como se puede observar en la gráfica, la mayoría de los alumnos proceden de los países de América Latina. Si contrastamos estos datos con la gráfica de población de Torrejón, veremos que la información coincide ya que la nacionalidad extranjera predominante en este municipio es la latinoamericana, seguida de la nacionalidad rumana. Al igual que ocurre en este colegio, los alumnos procedentes de Rumania ocupan el segundo lugar y, por último, los procedentes de Marruecos el tercero.

\section{- $\quad$ C.E.I.P El Olivar (San Fernando de Henares)}

El centro queda situado entre las calles de Nazario Calonge y Rafael Sánchez. La zona en la que se ubica el centro tiene un escaso número de inmigración con respecto al total de la localidad. Es una zona de reciente construcción, en el que predomina como tipo de vivienda,

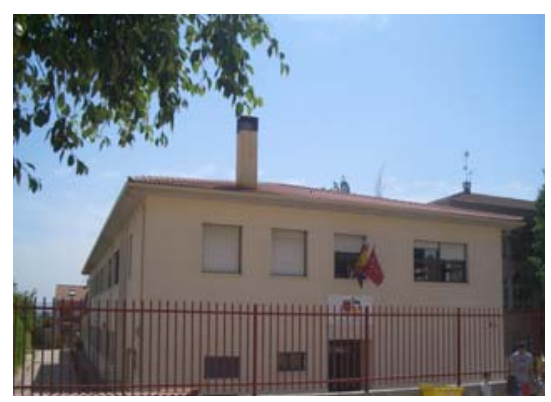
los chalets. El nivel socioeconómico es medio-bajo y no destacan grupo étnico en esta zona, por lo que las familias tienen una vida tranquila y pacífica. 


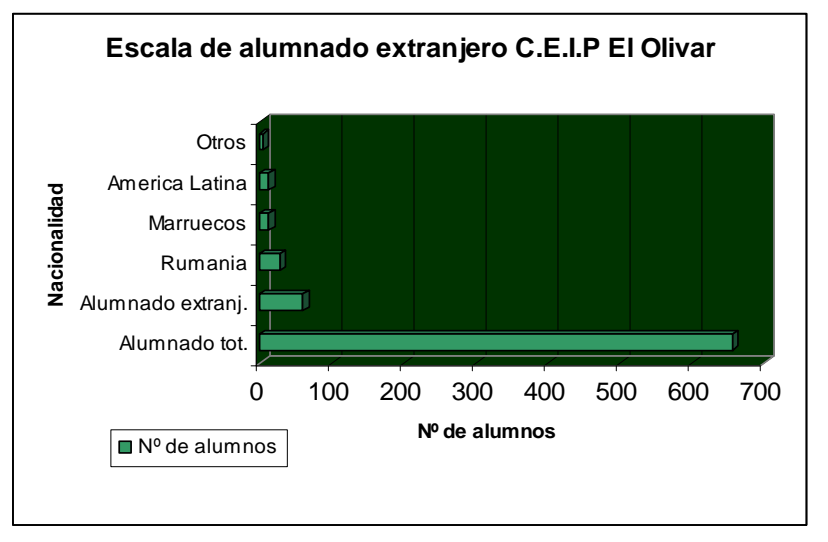

Como se puede observar en la gráfica, el alumnado extranjero en este centro escolar, es más bien escaso, ya que de los 655 alumnos que posee el colegio solo 80 son inmigrantes. Al igual que pasa con la escala de población inmigrante de San Fernando, la nacionalidad rumana es la que alberga mayor cantidad de alumnos, alcanzando casi la mitad del alumnado extranjero del centro. Les sigue la nacionalidad marroquí, y los alumnos procedentes de America Latina, siendo la nacionalidad ecuatoriana la que más alumnos alberga, y por último, otras nacionalidades de Europa del Este con un alumno polaco, tres moldavos y un búlgaro.

\section{Estado de la cuestión}

\section{Educación de la población inmigrante en el corredor del henares}

Desde los últimos diez años hasta la actualidad los centros educativos de toda la CCAA de Madrid han visto que el aumento de alumnos extranjeros es cada vez mayor, y si nos centramos en la zona Este de Madrid, veremos que cada vez son más los que se escolarizan en la educación pública, aunque en Educación Infantil, sobretodo en el primer ciclo, hay una fuerte demanda por parte de este grupo de población por los centros privados. Según el último informe de estadística de la Comunidad de Madrid, hemos recogido los siguientes datos:
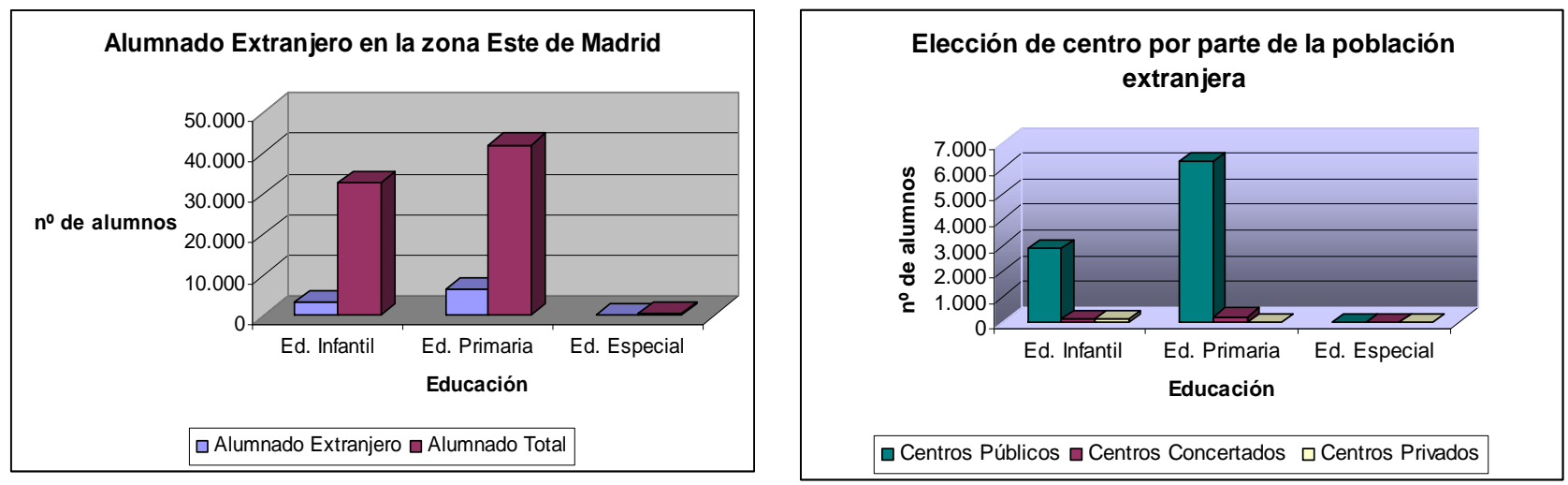

\section{Respuesta educativa a la población inmigrante en el corredor del henares}

- $\quad \underline{\text { Aula de Enlace }}$ 
Estas aulas están concebidas para atender a dos perfiles de alumnado extranjero:

- Alumnos extranjeros con necesidad de aprendizaje del español como lengua vehicular.

- Alumnos extranjeros con un grave desfase curricular como consecuencia de su falta de escolarización en el país de origen.

El número de estas aulas aumentan cada año, a medida que se incrementa el número de alumnos extranjeros. Entre los objetivos del aula de enlace esta la de facilitar la incorporación al Sistema Educativo Español y favorecer la integración de este en su entorno escolar y social.

La localización de las aulas de enlace dentro de los municipios de la zona Este de Madrid, son muchos los Colegios los que cuentan con un aula de enlace dentro del mismo centro. Cabe destacar en la localidad de San Fernando de Henares, el Colegio Público el Jarama. En Torrejón de Ardoz hay tres colegios con un aula de enlace, entre ellos: el Colegio Público Gabriel y Galán, El Andrés Segovia y el Juan Ramón Jiménez.

- Servicio de apoyo itinerante a alumnado inmigrante (S.A.I.)

Este servicio permite facilitar la incorporación educativa del alumnado inmigrante, especialmente cuando no domina el español. Realiza dos tipos de actuaciones:

- Servicio de orientación y asesoramiento. Permite asesorar sobre los programas de acogida que favorezcan la correcta inserción del alumnado inmigrante y la incorporación de la perspectiva intercultural al proceso educativo.

- Servicio de apoyo a centros: apoyo a la adquisición de competencias lingüísticas y comunicativas básicas del alumnado inmigrante de nueva incorporación.

- $\quad$ Servicio de Traductores e Intérpretes (SETI).

Es un servicio que se dedica a facilitar las relaciones de la familia con el centro escolar, debido al desconocimiento del castellano por parte de los miembros de la familia. El Servicio de Traductores e Intérpretes (SETI) se presta en más de 30 idiomas y sus funciones son las siguientes:

- Traducción de los documentos que son necesarios para la adecuada escolarización del alumnado inmigrante.

- Ejecución de labores de interpretación en las entrevistas o reuniones que se lleven a cabo desde cualquier servicio educativo.

El SETI incrementa sus actuaciones cada curso escolar. 


\section{Objetivos de la investigación}

Con esta investigación los objetivos que hemos querido desarrollar han sido los siguientes:

- Influencia de la presencia de alumnos extranjeros en las relaciones sociales en diferentes clases y en dos centros escolares.

- Conocer y analizar las medidas educativas que desarrolla cada centro para atender a la integración de estos alumnos.

- Efectividad de las medidas adaptadas en cada centro.

\section{Instrumentos utilizados para la investigación}

Los instrumentos que hemos utilizado para esta investigación son los siguientes:

- Sociogramas:

Hemos realizado un sociograma a dos clases de cada uno de los centros para estudiar las relaciones sociales dentro del aula, centrándonos especialmente en la aceptación de los alumnos inmigrantes dentro de cada grupo.

- Entrevista a los tutores:

El objetivo principal de estas entrevistas es el de conocer la opinión y recoger datos sobre los siguientes aspectos:

-Relaciones con los padres de los alumnos extranjeros.

-Relaciones sociales de los alumnos en clase.

-Opinión sobre las medidas de cada uno de los centros.

- Registros de observación:

Nuestro objetivo es estudiar una serie de comportamientos y actuaciones de los alumnos a través de una observación directa. Y ver de una forma más individualizada como se producen estos comportamientos en cada uno de los alumnos.

- Entrevista al director: 
Con este instrumento pretendemos recoger la información suficiente para interrelacionar cada uno de los elementos de la investigación. Y de esta manera sacar nuestras propias conclusiones.

\section{Análisis de los resultados obtenidos}

Análisis de los datos del sociograma

Hemos querido visualizar el porcentaje de alumnos de diferente nacionalidad en cada clase en donde hemos realizado esta investigación.

\section{COLEGIO EL OLIVAR (SAN FERNANDO DE HENARES)}
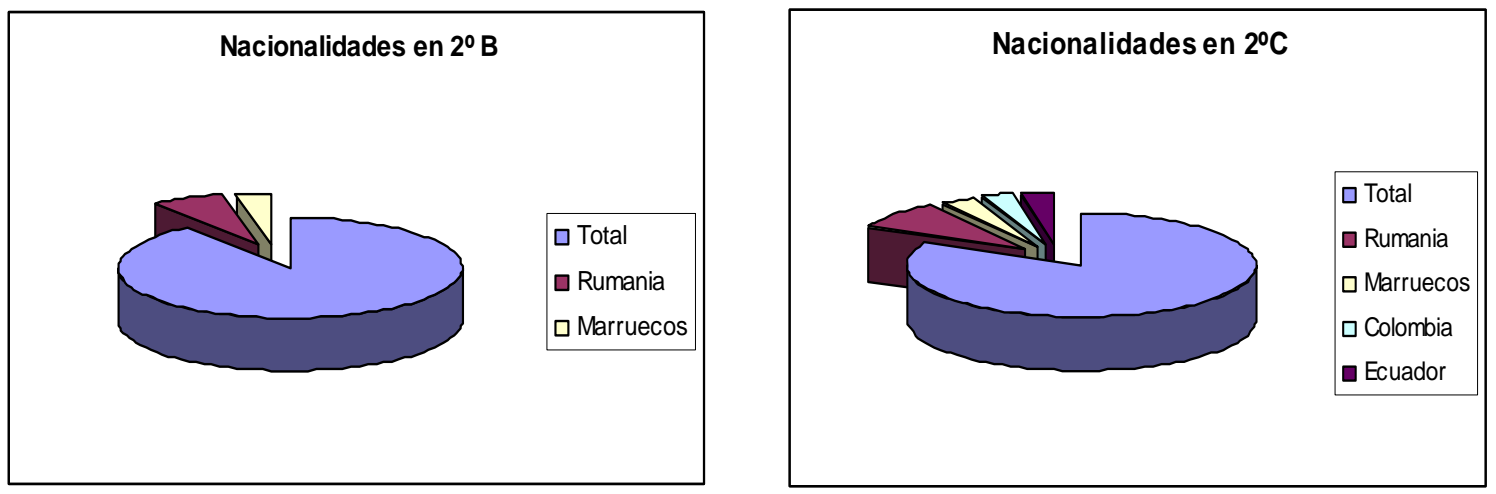

\section{COLEGIO SEVERO OCHOA (TORREJON DE ARDOZ)}
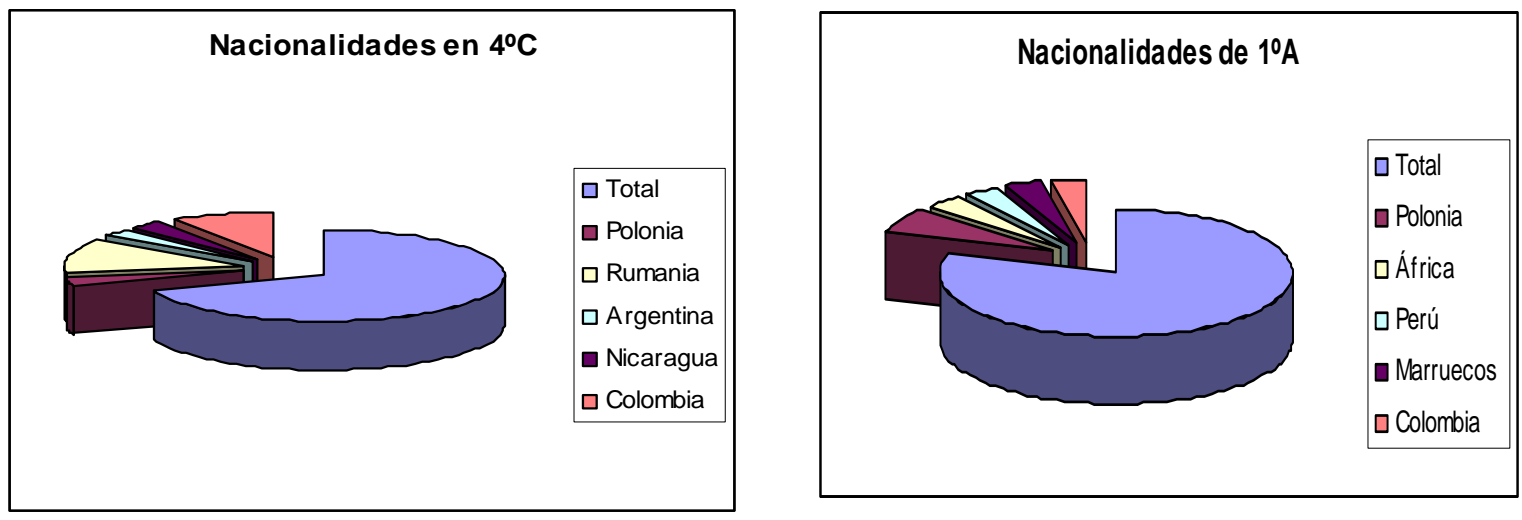

Antes de observar el análisis de las

gráficas debemos que tener en cuenta que estos datos se han realizado a partir de las preguntas realizadas a cada uno de los niños. Y en el que cada alumno ha tenido que elegir tan sólo a un/a compañero/a.

Los datos que reflejamos a continuación determinan que en centro escolar de Torrejón de Ardoz contiene un mayor porcentaje de inmigración y de diversidad cultural que el colegio El Olivar perteneciente a San Fernando. La nacionalidad predominante en las clases de El 
Olivar es la rumana, al igual que en la clase de $4^{\circ} \mathrm{C}$ del Severo Ochoa. Mientras que en la clase de $1^{\circ} \mathrm{A}$ predomina la polaca. En general, las nacionalidades de Europa del Este son las notables en estos centros.

\begin{tabular}{|c|c|c|c|c|c|}
\hline \multirow[t]{2}{*}{ C.E.I.P } & \multirow{2}{*}{$\begin{array}{c}\text { Alumnos } \\
\text { extranjeros en } \\
\text { el aula }\end{array}$} & \multicolumn{2}{|c|}{$\begin{array}{c}\text { Han salido elegidos a la pregunta: “ } \\
\text { Con quién te gusta jugar? }\end{array}$} & \multicolumn{2}{|c|}{$\begin{array}{c}\text { Han salido elegidos a la pregunta: "i } \\
\text { Con quién te gusta trabajar?" }\end{array}$} \\
\hline & & $\mathrm{N}^{\mathrm{o}}$ & $\%$ & $\mathrm{~N}^{\mathrm{o}}$ & $\%$ \\
\hline \multirow[t]{2}{*}{ "El Olivar" } & $2^{\circ} \mathrm{B}(3)$ & 0 & $0 \%$ & 0 & $0 \%$ \\
\hline & $2^{\circ} \mathrm{C}(6)$ & 1 & $15 \%$ & 3 & $50 \%$ \\
\hline \multirow[t]{2}{*}{ "Severo Ochoa" } & $1^{\circ} \mathrm{A}(6)$ & 4 & $66 \%$ & 4 & $66 \%$ \\
\hline & $4^{\circ} \mathrm{C}(10)$ & 6 & $60 \%$ & 7 & $70 \%$ \\
\hline
\end{tabular}

En el colegio El Olivar, observamos una cierta discriminación hacia los alumnos extranjeros, al contrario, que en el colegio Severo Ochoa. Comparando el porcentaje total de alumnos inmigrantes de los dos colegios, hemos observado que el colegio Severo Ochoa de Torrejón tiene un porcentaje mayor que el colegio El Olivar en San Fernando. Siendo el del Colegio Severo Ochoa de 21,75 \% y el del Olivar de 12,21\%, es decir, casi el doble.

Hemos analizado que la presencia de más alumnado extranjero en las aulas favorece las relaciones sociales entre ellos, y desaparecen las conductas discriminatorias. Hemos podido contrastar estos datos con el estudio y análisis de dos sociogramas realizados en cada centro.

En el colegio Severo Ochoa, uno de los sociogramas lo hemos efectuado en $4^{\circ}$ curso donde había mayor porcentaje de alumnos extranjero con respecto a los otros cursos. El segundo sociograma ha sido realizado en $1^{\circ}$ curso donde hay un menor porcentaje. Las conclusiones que hemos obtenido con estos dos cursos es que los niños de nacionalidad extranjera están bastante integrados en el conjunto de la clase. Y algunos de ellos han sido elegidos como los primeros por los otros niños para jugar o trabajar.

En el colegio El Olivar, los sociogramas han sido realizados en dos clases, $2^{\circ} \mathrm{B}$ y $2^{\circ} \mathrm{C}$, en el que una de ellas tiene un mayor número de alumnos extranjeros que en la otra. Una de las principales conclusiones que hemos obtenido es que en la clase en la que más alumnos de nacionalidad extranjera están más integrados entre sus compañeros ya que han sido elegidos en varias ocasiones. Sin embargo, en $2^{\circ} \mathrm{B}$ en la que solo hay tres inmigrantes, estos no han sido seleccionados en ninguna de los sociogramas.

Con estos datos podemos considerar que a mayor presencia de alumnos extranjeros en clase, hace que haya una mayor integración por parte del resto de los compañeros. Al igual que ocurre en el otro colegio. 
Análisis de las entrevistas a los tutores

Para esta investigación, se han realizado entrevistas a los tutores de las clases en las que estamos investigando. Los resultados que hemos obtenido, quedan reflejados en las siguientes gráficas:

Las relaciones con los padres de los alumnos extranjeros son muy buenas (1) o buenas (2), otro dice que es regular. Las relaciones de los alumnos extranjeros con sus compañeros y viceversa, son muy favorables. Dos profesores han dicho que son buenas, y los otros dos han dicho que son muy buenas. Se considera que dos profesores están de acuerdo con las medidas del centro, pero que se necesita más ayuda de especialistas. Mientras que los otros dos profesores tienen opiniones totalmente diferentes. Uno de ellos está totalmente de acuerdo, mientras que el otro profesor no esta de acuerdo con las decisiones que toma el centro.

En términos generales, todos los profesores tienen una buena relación con los padres de los alumnos, como vemos que ocurre en el colegio Severo Ochoa cuyas tutoras parecer tener una buena relación, aunque eso sí siempre con algunas excepciones. Sin embargo, en el colegio El Olivar, una de las profesoras ha tenido algún problema con los padres de dos alumnos de nacionalidad extranjera. "Regular, ya que he tenido problemas con los padres de dos de los alumnos extranjeros que hay en mi clase. Uno de los niños rumanos es hiperactivo. Siempre esta molestando a la clase y agita al resto, y yo percibo nerviosismo en el aula. [...]Le medicaron y trabajaba muy bien, pero un día su madre decidió quitarle la pastilla porque decía que ya estaba bien [...] hable con ella y no llegamos a ningún acuerdo. Con el padre del niño marroquí, no puedo hablar porque al ser mujer...ellos nos consideran inferiores”. Esta diferencia de opiniones entre los dos centros puede deberse a que en uno hay una mayor presencia de alumnado extranjero y hace que tanto los padres como los alumnos estén más integrados; sin embargo en el centro El Olivar al haber un número más reducido de inmigrantes una de las consecuencias es la falta de integración de los padres.

En lo que respecta a las relaciones de los alumnos extranjeros con el resto de la clase ambos centros coinciden siendo bastante buenas. Tanto en el colegio El Olivar como en el Severo Ochoa las relaciones sociales con los niños extranjeros suelen ser más fluidas y naturales cuando son más pequeños, ya que están conviviendo desde que entran al colegio y eso supone que no haya ningún tipo de discriminación por parte de sus compañeros. Aunque en dos de las clases hay niños que se encuentran solos con respecto a sus compañeros, normalmente estas situaciones solo suceden cuando el niño tiene algún trastorno del lenguaje y por lo tanto afecta a sus relaciones sociales (como es el caso de la clase de $1^{\circ} \mathrm{A}$ del Severo 
Ochoa) o algún problema como puede ser si es hiperactivo. "Son buenas, exceptuando al niño hiperactivo, porque como siempre esta molestando, el resto de la clase le coge manía”. En otros casos, la no aceptación del niño o niña en el aula se debe a la propia personalidad del alumno que a veces no es muy admitida por el resto de sus compañeros; en la clase de $4^{\circ} \mathrm{C}$ del Severo Ochoa un niño colombiano no está integrado debido a que está todo el rato molestando a sus compañeros y a la profesora y una niña rumana que tiene una aptitud de pasotismo ante los demás. "Se pasa el día molestando a sus compañeros y sobretodo a las niñas de la clase”.

En cuanto a las medidas que toma el centro para tratar los problemas del lenguaje todos los tutores coinciden en que se necesita mayor profesorado especializado para atender a estos niños correctamente, especialmente los profesores del Severo Ochoa. "Creo que las adaptaciones son correctas, pero el problema es que necesitamos mayor profesorado de Educación Especial, solo contamos con dos profesores de pedagogía terapéutica”. Aunque si hemos encontrado una cierta diferencia y es que uno de los profesores del colegio El Olivar parece estar completamente de acuerdo con las medidas que toma el centro en contraposición con otro profesor perteneciente al mismo centro que no comparte las mismas opiniones. "No estoy de acuerdo en que se meta a un niño en el aula sin saber nada de español porque para nosotros es una dificultad. Estas explicando en clase, y se quedan mirándote con cara de que no entienden nada".

Esta diferencia de opiniones puede deberse a que en el colegio El Olivar no hay un índice tan elevado de alumnado extranjero y por lo tanto, no se han encontrado con tantos casos como el colegio Severo Ochoa que cada año se encuentra con muchos alumnos con problemas con el lenguaje.

\section{Análisis documental}

- $\quad$ CEIP Severo Ochoa

-Propuestas organizativas adoptadas por el centro para atender al alumnado extranjero

Este colegio trabaja bajo las líneas del Proyecto de Atención a la Diversidad cuyo principal objetivo es fomentar unas relaciones sociales positivas y llenas de tolerancia. Estas medidas son llevadas a la práctica de forma gradual desde el primer ciclo hasta el tercer ciclo de Primaria.

Este Proyecto requiere de la participación de toda la Comunidad Educativa y se desarrolla en torno a los siguientes ejes de actuación: 
-Plan de Acogida: este plan realiza la recepción de los alumnos de nueva incorporación al centro y de sus padres. Sus funciones son: acogida del alumno y presentación de las instalaciones del centro, así como su adscripción al curso correspondiente y la integración del alumno en la clase y en el centro educativo.

Una vez que al alumno le es asignada una clase, se realiza un seguimiento del alumno para ver como es su integración con sus compañeros y en el propio centro, para ello se pone en marcha El Plan de Acción Tutorial.

El seguimiento se centra, como ya hemos citado en la integración del alumno, así como en la observación de la autonomía, responsabilidad, habilidades sociales del alumno, las relaciones entre familia y escuela y los apoyos que pudiera necesitar.

Si se detecta la necesidad de incorporar algún apoyo para ayudar al alumno o alumna, entonces se pone en marcha el Plan de Apoyos, cuyo objetivo primordial es apoyar mediante una actuación específica e individualizada el proceso de aprendizaje de aquellos alumnos con necesidades educativas especiales o de compensación educativa, así como de aquellos con retraso importante o dificultades en la superación de los objetivos básicos y significativos en las áreas de lenguaje o matemáticas.

Para detectar estos posibles apoyos se realiza una evaluación psicopedagógica y un seguimiento continuo durante la impartición de las diferentes áreas, con la finalidad de detectar en que área es necesario ese apoyo. Y finalmente se procede a adoptar unos criterios y unas medidas organizativas.

Por último, tras realizar una evaluación inicial para conocer su situación e informar a la Comisión de Escolarización y al profesorado del nivel y al Equipo de Orientación Educativa y Psicopedagógica (EOEP). Se actuará de forma diferente dependiendo de la edad del alumnado, en el caso de Educación Infantil entran directamente en el aula. En el caso de Primaria, tras la evaluación inicial se les asigna un curso y se pone en marcha un proyecto de compensatoria.

El proyecto de Educación compensatoria es un programa que destina los recursos específicos tanto materiales como humanos para garantizar el acceso, la incorporación e integración del alumnado con estas necesidades. De esta manera facilita la adquisición de los aprendizajes instrumentales básicos de Lengua y Matemáticas. Y procurar el conocimiento del castellano como medio de comunicación. Este proyecto presta especial atención a los niños con dificultades de tipo social o minorías étnicas o culturales que necesiten un apoyo debido a su incorporación tardía al sistema educativo, o en el caso de alumnos inmigrantes con desconocimiento del idioma. 
Los apoyos que se realizan se caracterizan por su flexibilidad y adaptación al alumnado, existen dos tipos de apoyos:

-Apoyos en grupos ordinarios: se realizan dentro de la clase, para reforzar los aprendizajes básicos en las áreas de Lengua Castellana y Matemáticas. Este apoyo lo reciben los alumnos que se encuentran en situación de desventaja social, quienes suelen presentar dos años de desfase curricular, pero sin embarbo, están bien integrados con el resto de la clase. Y aquellos alumnos con dificultades debidas al desconocimiento del castellano.

Este apoyo es desarrollado por un profesor/a que trabaja de forma conjunta con el profesor-tutor del aula.

-Grupos de apoyo: destinados a desarrollar actividades específicas relacionadas con la adquisición o refuerzo de aprendizajes básicos. Para ello, se forman grupos de apoyo fuera del aula de referencia durante una parte del horario escolar.

Para finalizar, el C.E.I.P Severo Ochoa no cuenta con aulas de enlace para los niños que desconocen nuestro idioma.

\section{-Adaptaciones curriculares individuales}

Son todos aquellos ajustes o modificaciones que se efectúan en los diferentes elementos de la propuesta educativa desarrollada por un alumno con el fin de responder a las necesidades educativas especiales.

Las adaptaciones significativas se realizan desde la programación, partiendo de la previa evaluación psicopedagógica, y que afectan a los elementos prescriptivos del currículo oficial por modificar objetivos generales de la etapa, contenidos básicos de las diferentes áreas curriculares y criterios de evaluación.

Estas adaptaciones pueden consistir en:

- Adecuar los objetivos, contenidos y criterios de evaluación.

- Priorizar determinados objetivos, contenidos y criterios de evaluación.

- Cambiar la temporalización de los objetivos y criterios de evaluación.

- Eliminar objetivos, contenidos y criterios de evaluación del nivel o ciclo correspondiente.

- Introducir contenidos, objetivos y criterios de evaluación de niveles o ciclos anteriores

Las adaptaciones curriculares individualizadas significativas realizadas para atender a las necesidades de los alumnos extranjeros, se centran principalmente en dos aspectos fundamentales: en primer lugar, los alumnos que tienen problemas con el idioma debido al desconocimiento de éste, y en segundo lugar, aquellos alumnos que presentan un retraso 
curricular debido a circunstancias personales y familiares (como la incorporación tardía al sistema educativo, o debido a factores culturales o fisiológicos).

Para aquellos alumnos que presentan un desconocimiento del castellano, las adaptaciones curriculares se centran principalmente en las áreas de Lengua Castellana y Matemáticas, ya que son las áreas impartidas en lengua castellana. En caso de Inglés y Conocimiento del Medio no existen adaptaciones curriculares, principalmente porque son impartidas en esta segunda lengua inglesa.

Estas adaptaciones se centran principalmente en la adecuación de los objetivos, contenidos y criterios de evaluación adaptados a su nivel de concreción curricular. Así como la priorización de aquellos objetivos básicos en cada una de las áreas y eliminación de aquellos de ampliación.

Como recursos materiales, el centro proporciona a los alumnos libros de texto y fichas didácticas adaptadas a sus necesidades.

La mayoría de los apoyos que reciben estos alumnos son dentro de los grupos ordinarios, siendo atendidos por un profesor de apoyo que trabaja de forma cooperativa con el profesor-tutor del aula.

Y en el caso de las adaptaciones curriculares desarrolladas a los alumnos que presentan un retraso curricular. Las adaptaciones tienen lugar tanto en las materias de Lengua Castellana y Matemáticas como en Lengua Inglesa y Conocimiento del Medio. Siendo su principal finalidad la adecuación, priorización, y eliminación de objetivos, contenidos y criterios de evaluación.

En algunas de las clases de Lengua Castellana y Matemáticas, estos alumnos salen a trabajar con el grupo de apoyo a otro aula, donde desarrollan actividades específicas para la adquisición de aprendizajes básicos.

- CEIP El Olivar

-Propuestas organizativas adoptadas por el centro para atender al alumnado extranjero.

Este centro escolar adopta las siguientes medidas, desarrolladas a lo largo de los siguientes proyectos que explicaremos a continuación:

En primer lugar este centro trabaja con el Proyecto de Educación Compensatoria, el cuál tendrá carácter preventivo y se regirá por el principio de normalización. El centro podrá adaptar parte del horario escolar que permita la atención del alumnado individualizada o en 
pequeño grupo para facilitar la adquisición de objetivos específicos dirigidos fundamentalmente al refuerzo de las técnicas instrumentales de lecto-escritura y cálculo.

La atención educativa será realizada conjuntamente por el profesorado tutor y el profesorado de apoyo, potenciando la adopción de fórmulas organizativas flexibles para responder a los diferentes intereses, motivaciones o necesidades de refuerzo que presente el alumno/a. Los objetivos principales ha desarrollar por este proyecto son los siguientes:

- Desarrollar la coordinación necesaria entre los profesores - tutores y el profesor de Ed. Compensatoria para elaborar las correspondientes Adaptaciones Curriculares.

- Fomentar la coordinación entre escuela, familias y las diversas instituciones relacionadas con estos niños.

- Crear hábitos de higiene, salud y nutrición.

- Favorecer su inserción socio-educativa respetando su propia identidad para lograr una rápida integración.

En segundo lugar el centro dispone del servicio de Audición y Lenguaje, en el que el maestro especialista centra su labor en todo lo relacionado con la comunicación y el lenguaje de los alumnos, sobre todo en aquéllos que necesiten de una ayuda especial. Las funciones del maestro de Audición y Lenguaje son:

- Atención directa a todos los alumnos que presenten dificultades, tanto en el desarrollo del lenguaje y/o la comunicación.

- Proporcionar tratamiento o reeducación en los posibles trastornos que aparecen en este área.

- Servir de apoyo y orientación al resto de los profesores del centro, a la hora de realizar las adaptaciones curriculares oportunas, para poder ofrecer así una respuesta educativa ajustada a las necesidades educativas especiales de cada uno de los alumnos.

Además para aquellos alumnos que se encuentren entre $3^{\circ}$ y $6^{\circ}$, se les destina al Aula de enlace durante3 meses. Debido al reducido número de alumno inmigrante en el centro, El Olivar no dispone de ningún aula de enlace, por lo que son enviados al C.E.I.P El Jarama que si dispone de este tipo de aula. Una vez finalizada esta etapa, los alumnos vuelven a su clase pero con salidas al aula de Compensatoria.

De Infantil a $2^{\circ}$ curso de primaria hay una inmersión lingüística, en el que se introduce al alumno en el aula como al resto. Una de las medidas que toma el centro es crear aulas heterogéneas para tener la posibilidad de integrar y traducir (en la medida de lo posible). 
Además se les administrarán clases de refuerzo con profesores de apoyo y la intervención de los especialistas de Audición y Lenguaje.

\section{-Adaptaciones curriculares individuales}

Los objetivos generales llevados a cabo en las adaptaciones curriculares significativas por este centro son los siguientes:

- Dominio oral y escrito de la lengua castellana.

- Acceso al mismo currículo que sigue el resto del alumnado.

- Logro de una progresiva autonomía personal dentro del ámbito escolar y social.

Para ello se tomarán las siguientes medidas:

- Realizar un diagnóstico de las necesidades educativas y lingüísticas del alumnado.

- Facilitar una rápida y adecuada escolarización del alumnado inmigrante, en los mismos términos que el autóctono.

- Atender las necesidades educativas y lingüísticas personales mediante refuerzos individuales y/o colectivos o la adaptación curricular.

- Inducir a los miembros del grupo autóctono para que acepten como iguales al alumnado inmigrante.

- Facilitar la integración de las diferencias culturales potenciando el respeto y el mutuo conocimiento de las mismas

Las adaptaciones curriculares significativas van destinadas a aquellos alumnos inmigrantes que presentan un desfase curricular. Al ser trasladados al aula de enlace del otro colegio los alumnos que presentan problemas con el idioma, por ello no se realiza ninguna adaptación curricular de este tipo.

Los alumnos que presentan desfase curricular, en cuanto a los materiales, cuentan con un material específico como libros de texto o fichas que son trabajados en clase con el resto de compañeros. Los apoyos se realizan dentro del grupo ordinario sin sacar al alumno del aula y es ayudado por un profesor de apoyo. Sin embargo, en las áreas de Lengua Castellana y Matemáticas, los alumnos trabajan con el profesor de apoyo fuera del aula.

Los centros educativos investigados no nos han facilitado toda la información necesaria así como la consulta de documentos clave como la PGA y PEC. Por lo que prácticamente nos hemos basado en las entrevistas y nuevas tomas de contacto con los directores del Centro. 
$\mathrm{Al}$ comparar las medidas que adopta cada centro educativo, vemos que estas presentan ciertas similitudes, y a la vez grandes diferencias. Las similitudes se encuentran en que ambos centros ponen en práctica un plan de compensatoria en el que el alumno realiza los apoyos fuera del aula. Y la diferencia la encontramos en que el centro El Olivar cuenta con un aula de enlace, aunque no esté ubicada en el mismo centro, mientras que el colegio Severo Ochoa no dispone de esta medida. Con lo cual las adaptaciones curriculares referidas al desfase curricular se asemejan bastante, ya que emplean el mismo tipo material.

Análisis de los registros de observación.

- $\quad$ Análisis de las clases $2^{\circ} \mathrm{B}$ y $2^{\circ} \mathrm{C}$ del C.E.I.P. El Olivar

En general en $2^{\circ} \mathrm{B}$ los tres niños suelen participar en las actividades realizadas en clase, pero vemos que sólo uno de ellos atiende en clase con frecuencia. Pero aún así necesita que le expliquen los contenidos.

Normalmente todos los alumnos necesitan ser ayudados y en pocas ocasiones entienden los contenidos.

En $2^{\circ} \mathrm{C}$ todos los niños atienden y suelen participar con frecuencia. Sin embargo vemos que mitad o menos de la mitad normalmente no entienden los contenidos y por lo tanto necesitan ser ayudados.

\section{- $\quad$ Análisis de las clases $4^{\circ} \mathrm{C}$ y $1^{\circ}$ A del C.E.I.P. Severo Ochoa}

En la clase de $4^{\circ} \mathrm{C}$ la mayoría de los alumnos atienden a las actividades puestas en práctica en clase, pero sin embargo sólo la mitad de ellos participa frecuentemente.

Más de la mitad de la clase entiende los contenidos y como consecuencia de ello, un grupo reducido es el que acaba necesitando ayuda.

En la clase de $1^{\circ} \mathrm{A}$ ocurre lo mismo que en el grupo anterior en el que casi todos los alumnos atienden aunque con alguna excepción. En cuanto a la categoría de participación podemos destacar que dos niñas son las que siempre participan. En cuanto a la adquisición de contenidos sólo un grupo reducido de la clase son quiénes los alcanzan, coincidiendo con los mismos alumnos que participan. Y por lo tanto, la gran mayoría son los que necesitan ayuda para entenderlos.

Tras el análisis realizado en ambos centros educativos hemos llegado a la conclusión de que la gran mayoría de los alumnos, a pesar de que atienden y suelen participar en clase no llegan a alcanzar los contenidos propuestos. Y por lo tanto, necesitan una ayuda constante para compensar sus dificultades de aprendizaje. 


\section{- $\quad$ Comparación de las clases de los dos centros educativos}

Vemos que los alumnos extranjeros de ambos centros educativos a excepción de la clase de $2^{\circ} \mathrm{B}$ suelen atender en clase. Aunque vemos que en los dos colegios los alumnos no llegan a entender los contenidos propuestos y por lo tanto, la gran mayoría necesita un apoyo complementario por parte del profesor.

Hemos observado que los alumnos del colegio El Olivar son más participativos que los alumnos del Severo Ochoa.

\section{Conclusiones generales}

Tras haber estudiado parcialmente varios aspectos los cuales hemos interrelacionado entre sí. Llegamos a las siguientes conclusiones:

- Las medidas que toma cada uno de los centros respecto a la integración de los alumnos extranjeros en las aulas son suficientes, aunque luego la puesta en práctica no son tan eficientes como deberían. En el colegio El Olivar al haber menor número de alumnos extranjeros se produce una cierta discriminación hacia ellos. Y sin embargo, en el colegio Severo Ochoa en líneas generales hay una buena integración al haber un mayor porcentaje de alumnado extranjero que el anterior centro.

- Las opiniones de los maestros del colegio EL Olivar reflejan una cierta diferencia con la realidad de la clase, ya que creen que las relaciones de los alumnos extranjeros con los demás compañeros son bastante aceptables. En cambio, los datos obtenidos del sociograma nos dicen todo lo contrario, existiendo cierto rechazo a alguno de los alumnos. Sin embargo, en el colegio Severo Ochoa los profesores parecen ver más realidad del centro. Siendo una de las profesores la que reconocía que dos de los alumnos no están integrados en la clase.

- Gracias a la ayuda de las tablas de observación hemos podido concluir que las medidas y recursos que adoptan los dos centros son insuficientes ante las necesidades y problemas de lenguaje y de conocimientos, ya que la ayuda que necesitan estos alumnos es constante. Por lo tanto los centros deberían contar con una plantilla más grande de profesores de apoyo y especialistas sobretodo de Audición y Lenguaje.

- Todos los profesores de los dos centros excepto uno perciben la realidad con la que se convive día a día en las aulas, mostrándose en desacuerdo con las medidas tomadas 
por sus centros. Ya que todos coinciden en la necesidad de la presencia de más apoyo dentro y fuera de las clase.

- Tras las observaciones y conclusiones realizadas, queremos destacar que la presencia de un número elevado de alumnos extranjeros no incide negativamente en el proceso de E-A.

- Como reflexión final y personal, creemos que las medidas tomadas en el centro Severo Ochoa no son todo lo eficientes que tendrían que ser, ya que no existe aula de enlace y los alumnos pasan directamente a su aula definitiva con un programa de compensatoria. Creemos que el aula de enlace es un elemento imprescindible ya que permite que el alumno evolucione de una manera más rápida y tenga una atención más individualizada para que de esta manera pueda incorporarse al aula sin problemas.

- Al no ocurrir esto en el centro de Torrejón de Ardoz, los alumnos son atendidos por un profesor de compensatoria en horas fuera de clase, y por un profesor de apoyo dentro de la clase ordinaria. De esta manera, los alumnos que salen del aula quedan desorientados con respecto a sus compañeros.

\section{A STUDY ABOUT IMMIGRATION AND INTEGRATION OF STUDENTS IN TWO SCHOOLS IN MADRID, SPAIN}

ABSTRACT: This investigation project is based immigration at the schools, due to in the last few years the number of foreign pupils has markedly increased. The research has been made in two educational centers belong to Corredor de Henares area, concretely in four classes with a different Primaria levels. These schools show a big different at the percent of the foreign students registered. Doing this project we have wanted to focus in the following points tostudy: Firstly, the social relationships with the end of knowing the integration level of the foreign students compare to the rest of the students. Next we have researched the measures taken by the two centers for the integration and adaptation of those pupils to the school and educational system. And finally we have analized how these measures adjust to the foreign students' situation.

KEYWORDS: Inclusion. Integration. Adaptation. Measures for student's. Immigration. "Linked classroom”.

\section{REFERENCIAS}

AJA, E. et al. La inmigración extranjera en España: los retos educativos. Barcelona: Fundación La Caixa, 2000. (Estudios Sociales, 1). 
CONSEJERÍA DE EDUCACIÓN. Secretaría General Técnica: 2006-2007. Estadística de las enseñanzas de régimen general, especial y personas adultas. Disponible en:

<http://www.madrid.org/ ESTADISTICA-1.pdf>. Acceso en: 19 mayo 2008.

MINISTERIO DE EDUCACIÓN Y CIENCIA. Centro de Investigación y Documentación Educativa [CIDE]. La atención al alumnado inmigrante en el sistema educativo España. 2005. Disponible en: <http://www.educacion.es/

cide/espanol/publicaciones/colecciones/investigacion/col168/col168pc.pdf>. Acceso en:

Acceso en: 19 mayo 2008.

DIRECCIÓN GENERAL DE PROMOCIÓN EDUCATIVA. Consejería de Educación y Comunidad de Madrid. Atención a la Diversidad en la Comunidad de Madrid. 2006. Disponible en : <www.pdf.obrasocial.comunicacions.com/es/esp/es01_esp.pdf>. Acceso en: Acceso en: 19 mayo 2008.

\section{BIBLIOGRAFÍA CONSULTADA}

GALVE, J. L.; SEBASTIÁN, E. Las medidas de atención a la diversidad. In: MARTÍN BRIS, M.; MARGALEF, L. La educación para la diversidad: múltiples miradas. Alcalá de Henares: Servicio de Publicaciones de la Universidad de Alcalá, 2000. p.123-166.

SEBASTIÁN, E. La adaptacion curricular individual como estrategia de la escuela inclusiva. DOXA: revista paulista de psicologia e educação, Araraquara, v.12, n.2, p.9-26, 2008.

Escuela inclusiva: ideas para ponerla en práctica. Revista Servicio Social y Realidad, v.16, n.2, p. 111-121, 2007 .

Las adaptaciones curriculares de pequeno porte o no significativas en el contexto

brasileño. Revista Ibero-Americana de Estudos em Educação, Araraquara, v.2, n.2, 2007b. Disponible en : <http://seer.fclar.unesp.br/index.php/iberoamericana/article/view/463>. Acceso en: Acceso en: 19 mayo 2008.

A escola inclusiva: bases legais para sua organização. Rev Educatio, Murcia, n.45, p.10-15, mayo 2005.

El plan de atención a la diversidad. Rev Clasè, México, n.13, dic. 2003a.

La planificación de la atención a la diversidad. In: MARTÍN BRIS, M. (Coord.). Planificación educativa: intercambio de experiencias y perspectivas. Alcalá de Henares: Servicio de Publicaciones de la Universidad de Alcalá, 2003b. p.29-46.

Adaptación curricular individualizada: modelo alumno superdotado. In: GALVE, J. L.; TRALLERO, M. Las adaptaciones curriculares. Casos Prácticos. Madrid: CEPE, 2002a. 
Adaptación curricular individualizada: modelo cataluña. In: GALVE, J. L.;

TRALLERO, M. Las adaptaciones curriculares: educación infantil, primaria y secundaria. Madrid: CEPE, 2002b.

SEBASTIÁN, E.; AYALA, C. L. Modelo para una programación coherente de los ámbitos. In: AA.VV. II Encuentro de Diversificación. Coslada: CPR de Coslada, 2001.

SEBASTIÁN, E. GALVE, J. L. Atención a la diversidad en el aula: programa de diversificación curricular. In: MARCHENA, R.; DOMINGO, J. (Coord.) De la integración a una educación para todos: la atención a la diversidad desde la educación primaria a la universidad. Madrid: CEPE, 2002. p. 103-110.

VÁSQUEZ GONZÁLEZ, L. Inmigración en el Corredor de Henares: resumen ejecutivo. Madrid: CIAES, 2007, 